\title{
Optical interferometry between image-bearing beams and their redirected phase conjugates
}

\author{
Mordechai Segev and Amnon Yariv \\ California Institute of Technology, Pasadena, California 91125
}

Received July 26, 1991

\begin{abstract}
We present three new interferometers, which are based on interference between combinations of image-bearing optical fields and their redirected phase-conjugate reflections in two- and three-dimensional configurations. Our interferometers can be used to implement mathematical operations on images, including the Hartley transform.
\end{abstract}

Optical interferometers are commonly used for a variety of applications, including spectral analysis, displacement measurements, and gyroscopes. They were also proposed, in a more limited sense, for applications involving image processing. ${ }^{1}$ In what follows we describe three new interferometers that implement mathematical operations on images borne by coherent input laser beams. Each interferometer executes a different operator of interest in image processing.

The first interferometer implements the twodimensional Hartley transform of a function $f(x, y, z)$. The Hartley transform ${ }^{2}$ is defined by

$$
H(u, v, z)=\int_{-\infty}^{\infty} \int_{-\infty}^{\infty} f(x, y, z) \operatorname{cas}[2 \pi(u x+v y)] \mathrm{d} x \mathrm{~d} y,
$$

where $\operatorname{cas}(\alpha) \equiv \cos (\alpha)+\sin (\alpha)$. This $(H)$ transform was first suggested by $\mathrm{Hartley}^{3}$ and proposed for computations in the fields of pattern recognition and image processing by Bracewell., ${ }^{4,5}$ Its main advantages over the Fourier transform are (i) if $f(x, y, z)$ is real on a plane of a constant $z$, then $H(u, v, z)$ is real, and (ii) the $H$ transform and its inverse transform are identical. The first property is important for applications in both computer and optical image processing of real images. In the first case it saves memory and reduces the computation time, and in the optical case it may suggest realization of real matched filters, with no need for encoding phases (of the complex Fourier transform of a general real image). Nevertheless, while the computerized calculation of the $H$ transform is relatively simple, the optical realization is problematic. ${ }^{6,7}$ For our first interferometer we present, among other operations on the input image, a simple realization of the $H$ transform.

Our first interferometer is shown in Fig. 1. It is based on redirection properties of optical images in three-dimensional systems. The input image $f(x, y, z=0)$ is split by the beam splitter $\left(\mathrm{BS}_{1}\right)$ into the two separate arms of the interferometer. The transmitted beam is simply directed forward by the ordinary mirror $\mathrm{M}$ and the piezoelectric mirror $\mathrm{PZ}$.
The reflected image from $\mathrm{BS}_{1}$ is redirected by periscope $P_{1}$, brought to an upper level, and rotated by $90^{\circ}$. The resulting field past the first periscope is $f(-y, x, z)$. This beam is then redirected again by the second periscope $\mathrm{P}_{2}$, lowered to the original level, and rotated by $90^{\circ}$, which yields $f(-x,-y, z)$. (Note that the two periscopes in the same arm not only returned the beam to the original plane but also inverted it in both the $x$ and the $y$ directions.) This beam is combined with the first arm through reflection from the second beam splitter $\mathrm{BS}_{2}$. The piezoelectric mirror in the first arm is used to adjust the optical path difference between the arms. In the most general case it introduces an arbitrary phase shift of $\varphi$ of one arm with respect to the other. The combined image-bearing beams propagate through lens $L$ and are Fourier transformed at the output $O$ plane. The image at this plane is

$$
O(u, v)=F(-u,-v)+\exp (i \varphi) F(u, v),
$$

where $F(u, v)$ is the two-dimensional Fourier transform of $f(x, y, z=0)$. In a special case, where $f(x, y, z=0)$ is real and $\varphi$ is adjusted to be $\pi / 2$, the result is the $H$ transform with an additional factor ${ }^{6}$

$$
O(u, v)=\frac{\exp (i \pi / 4)}{\sqrt{2}} H(u, v) .
$$

A demonstration of our $H$ transformer is given in Fig. 2. A real image $f(x, y, z=0)$ was obtained by collimation of an expanded laser beam and propagation through an image (the letter $\mathrm{R}$ )-bearing transparency. The object plane of lens $\mathrm{L}$ (at the output of the interferometer) is given in Fig. 2(a). For comparison with Ref. 6, we show in Fig. 2(b) the $H$ transform (obtained by our $H$ interferometer) of a circular slit, which is shifted from the origin $(x=0$, $y=0$ ). It displays Airy rings (the Fourier transform of the circular slit) modulated by linear fringes. The period of the fringes corresponds to the distance from the center of the shifted slit to the origin, and their orientation indicates the displacement direction. ${ }^{6}$

The use of the object plane of lens $\mathrm{L}$ in our $H$ interferometer suggests other applications through 


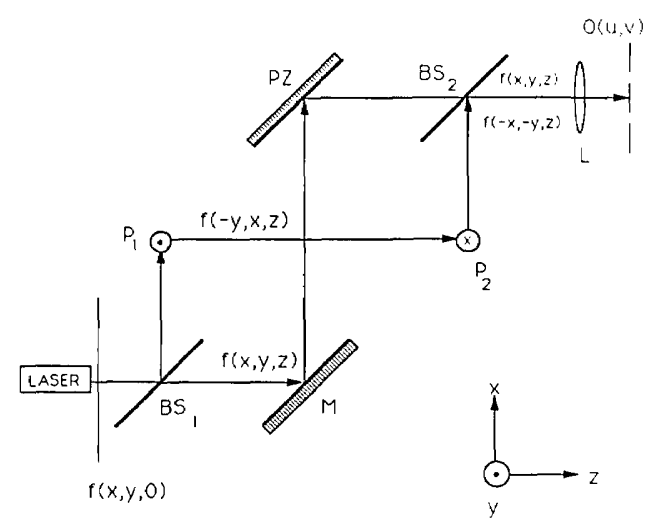

Fig. 1. Scheme of the three-dimensional periscopic $H$ interferometer. The input and output functions are $f(x, y, 0)$ and $O(u, v)$, respectively.

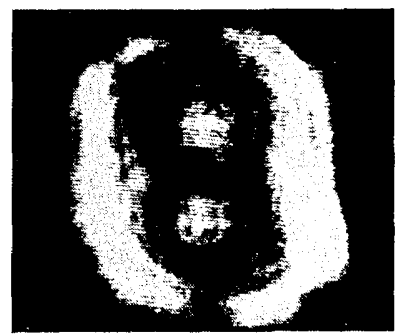

(a)

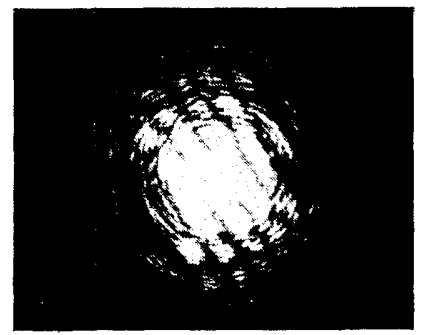

(b)
Fig. 2. Experimental results obtained with the interferometer shown in Fig. 1. (a) The object plane of lens $L$ for an input image of the letter $\mathrm{R}$. (b) The $H$ transform of a displaced circular slit obtained at the focal plane of lens $\mathrm{L}$.

its mathematical operation on the input image, $f(-x,-y, z=0)+\exp (i \varphi) f(x, y, z=0)$. When $\varphi=\pi(\varphi=0)$ we get subtraction (addition) between the original image and its reflection by the origin $(x=0, y=0)$. This property is useful to extract the even (odd) parts of a given input image and is described by the following:

$$
\begin{aligned}
& f_{e}(x, y, z) \equiv 0.5[f(x, y, z)+f(-x,-y, z)], \\
& f_{o}(x, y, z) \equiv 0.5[f(x, y, z)-f(-x,-y, z)],
\end{aligned}
$$

where $f_{e}$ and $f_{o}$ are the even and odd symmetry parts of $f$, respectively. This operation is valid for a general input image $f(x, y, z)$, which may be complex in the most general case.

Our second interferometer (the so-called $\mathrm{S}$ interferometer) involves interference between an image-bearing input beam and its redirected phaseconjugate reflection in a planar system. Consider the configuration of Fig. 3 and a general (complex) input image $f(x, y, z)$. The image-bearing beam is split by the first beam splitter $\mathrm{BS}_{1}$. The transmitted portion is incident upon a phase-conjugate mirror (PCM), which was realized by a passive (semilinear ${ }^{8}$ ) photorefractive $\mathrm{BaTiO}_{3}$ oscillator. The phase-conjugate reflection $f^{*}(x, y, z)$ is now propagating backward (in the $-z$ direction). We redirected this phase-conjugate beam forward by three mirrors $\left(\mathrm{BS}_{1}, \mathrm{M}_{1}\right.$, and $\left.\mathrm{M}_{2}\right)$ and combined it with the original unconjugated input at the second beam splitter $\mathrm{BS}_{2}$. It can be shown straightforwardly that the redirection by three mirrors does not invert the image in the $x$ and $y$ directions. The output of the interferometer, at the Fourier plane of lens $L$, is the superposition

$$
S(u, v)=F^{*}(-u,-v)+\exp (i \varphi) F(u, v) .
$$

This transform may be useful in a variety of applications, of which two are of special interest. The first application is extraction of the real and imaginary parts of a complex input image and their Fourier transforms. The input image can be expressed by $f(x, y)=f_{1}(x, y)+i f_{2}(x, y)$, where $f_{1}$ and $f_{2}$ are real functions, and their Fourier transforms satisfy $F_{j}^{*}(-u,-v)=F_{j}(u, v)$ (for $\left.j=1,2\right)$. The interferometer output gives

$$
\begin{aligned}
S(u, v)= & 2 \exp (i \varphi / 2)\left[F_{1}(u, v) \cos (\varphi / 2)\right. \\
& \left.+F_{2}(u, v) \sin (\varphi / 2)\right] .
\end{aligned}
$$

Adjustment of the phase $\varphi$ to be $0(\pi)$ gives $F_{1}\left(F_{2}\right)$ at the output plane. A similar operation on the input image can be implemented in the object plane of the lens and can separate $f_{1}(x, y)$ from $f_{2}(x, y)$.

The second application is accurate phase detection. Consider the case of a complex input image, which can be expressed by

$$
f(x, y)=f_{R}(x, y) \exp [i \theta(x, y)],
$$

where $f_{R}$ and $\theta$ are real functions. Using Eq. (7) in the $\mathrm{S}$ interferometer, we obtain at the object plane of lens L

$$
\begin{aligned}
f^{*}(x, y)+\exp (i \varphi) f(x, y)= & 2 f_{R}(x, y) \exp (i \varphi / 2) \\
& \times \cos [\theta(x, y)+\varphi / 2],
\end{aligned}
$$

where $\varphi$ is the phase delay. For a known $f_{R}$, detection of the phase becomes sensitive. For example, consider an image-bearing Gaussian beam, where the input plane is located at a small distance $z$ from

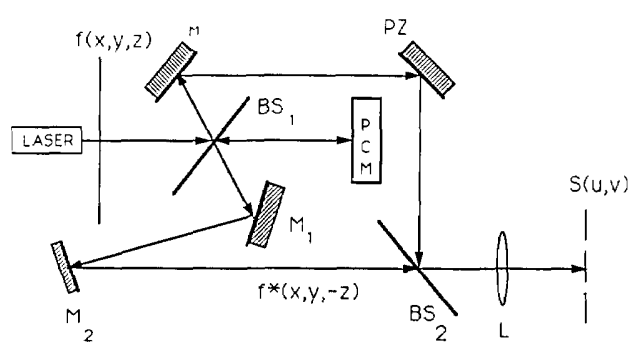

Fig. 3. Scheme of the $\mathrm{S}$ interferometer.

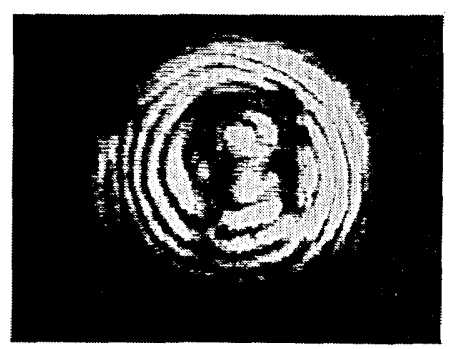

Fig. 4. Experimental results obtained at the object plane of the lens at the output of the $S$ interferometer of Fig. 3 for an input image of the letter $R$. 


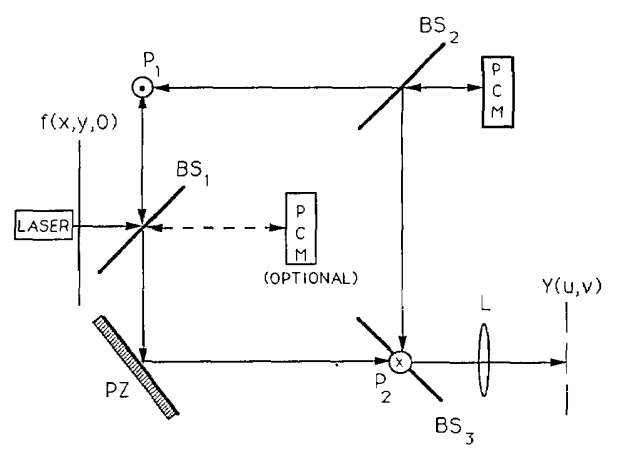

Fig. 5. Scheme of the Y interferometer. The dashed line and the additional PCM are optional.

the waist. The displacement $z$ introduces a spherical phase of radius $R(z)$. If waist $\omega_{0}$ is sufficiently large, then $z \ll z_{0}$, and $R(z)$ is large. Detection of this spherical phase is automatically done in the $\mathrm{S}$ interferometer, through the interference between the two beams with radii of curvature of opposite signs (the original input and its phase conjugate). The sensitivity of this interferometer is double the sensitivity of conventional interferometry, and the fringe contrast is always unity, regardless of the pictorial information $f_{R}(x, y)$. We note that this property was demonstrated in a previous study ${ }^{9}$ that used conventional holography to make the PCM.

The last arguments are demonstrated in Fig. 4. The input, of a Gaussian beam bearing the letter R, was introduced by a transparency located at a distance $z \ll 1 \mathrm{~cm}$ from the waist (the waist size $2 \omega_{0}$ was $1 \mathrm{~cm}$, i.e., the Gaussian beam was collimated). The object plane of the lens $\mathrm{L}$ at the interferometer output is shown in Fig. 4. The superposition of the input and the phase-conjugate beams is modulated by circular fringes that result from the interference between the input diverging beam and its redirected phase-conjugate reflection and indicates the radius of curvature of the Gaussian input beam, which in return gives the distance from the waist $z$.

Our third interferometer is a combination of the first two. It involves interference between two redirected phase-conjugate reflections of the same input image, each inverted in one direction. Consider the configuration in Fig. 5. The image-bearing input beam $f(x, y, z=0)$ is split by the beam splitter $\mathrm{BS}_{1}$. The reflected beam is brought to an upper level and rotated by $90^{\circ}$ by the first periscope $P_{1}$. This beam is reflected from a PCM, and the reflection $f^{*}(-y, x, z)$ is fed, after being split by $\mathrm{BS}_{2}$, into the two interferometer arms. The transmitted portion propagates all the way back to $\mathrm{BS}_{1}$ and is redirected forward by the piezoelectric mirror PZ. The use of two mirrors only (PZ and the combined operation of the periscope $\mathrm{P}_{1}$ mirrors) for the redirection operation inverts the image in the $x$ direction, which results in $f^{*}(-x, y, z)$. The second portion of the phase-conjugate beam is reflected from $\mathrm{BS}_{2}$, lowered to the original level, and redirected forward by the periscope $P_{2}$. This time the redirection operation is made in the $y$ direction, again by two mirrors only, which yields inversion of the image to $f^{*}(x,-y$, $z=0$ ). The two beams are recombined by beam splitter $\mathrm{BS}_{3}$, which doubles as the lower mirror of periscope $\mathrm{P}_{2}$, and Fourier transformed by lens $\mathrm{L}$. The result at the focal plane of the lens is

$$
\hat{Y}(u, v)=F^{*}(u,-v)+\exp (i \varphi) F^{*}(-u, v) .
$$

Equation (9) represents a new linear transform on a general input image $f(x, y, z)$ that may be useful for applications in optical image processing. One special case is when the input image $f(x, y, 0)$ is real and the phase delay $\varphi$ is adjusted to be $\pi / 2$. In this case, the so-called Y transform is defined by

$$
\begin{aligned}
Y(u, v) & =\int_{-\infty}^{\infty} \int_{-\infty}^{\infty} f(x, y, z) \operatorname{cas}[2 \pi(u x-v y)] \mathrm{d} x \mathrm{~d} y \\
& =\sqrt{2} \exp (i \pi / 4) \hat{Y}(u, v) .
\end{aligned}
$$

The transform operation is then identical to the output of the interferometer (with an additional phase factor). We note the following: (i) The Y transform is an antisymmetric (with respect to the transverse directions $x$ and $y$ ) version of the ordinary Hartley transform. (ii) For better matching of the optical path between the two interferometer arms in Fig. 5, we added an additional phase-conjugate mirror on the transmitted portion through beam splitter $\mathrm{BS}_{1}$ [which yields the same $f^{*}(x,-y, z=0)$ at the object plane of lens L] and used its reflection instead the original one. (iii) The Y transform can also be achieved by simply replacing the PCM by an ordinary mirror. (iv) The PCM realization offers another mathematical operation on the input image, obtained at the object plane of lens $\mathrm{L}, f^{*}(x,-y)+$ $\exp (i \varphi) f^{* *}(-x, y)$.

In conclusion, we have presented three new interferometers that utilize three-dimensional and phaseconjugate optics as a means of coherent image processing. Our interferometers offer implementation of various mathematical operations on images. Some correspond to known transforms, and some are suggested here for the first time to our knowledge.

We acknowledge useful discussions with Joseph Rosen of the Technion-Israel Institute of Technology, Haifa, Israel, and with Richard Solomon of the California Institute of Technology. This research was sponsored by the U.S. Army Research Office, Durham, North Carolina, and the U.S. Air Force Office of Scientific Research.

\section{References}

1. E. Wolf, Progress in Optics (Elsevier, Amsterdam, 1987), Vol. 24, pp. 132 and 458, and references therein.

2. J. Villasenor and R. N. Bracewell, Nature (London) 330, 735 (1987).

3. R. V. L. Hartley, Proc. Inst. Radio Eng. 30, 144 (1942).

4. R. N. Bracewell, J. Opt. Soc. Am. 73, 1832 (1983).

5. R. N. Bracewell, Proc. IEEE 72, 1010 (1984).

6. R. N. Bracewell, H. Bartelt, A. W. Lohmann, and N. Streibl, Appl. Opt. 24, 1401 (1985).

7. Y. Li and G. Eichmann, Opt. Commun. 56, 150 (1985).

8. J. O. White, M. Cronin-Golomb, B. Fischer, and A. Yariv, Appl. Phys. Lett. 40, 450 (1982).

9. Y. Fainman, E. Lenz, and J. Shamir, Appl. Opt. 20, 158 (1981). 